\title{
INFRARED EMISSION FROM PHOTO-EXCITED GASEOUS BENZENE: DETECTION WITH A NEW HOME-MADE SPECTROMETER
}

\author{
G. Féraud ${ }^{1}$, Y. Carpentier ${ }^{2}$, T. Pino ${ }^{1}$, Y. Longval ${ }^{3}$, E. Dartois ${ }^{3}$, \\ T. Chamaillé ${ }^{1}$, R. Vasquez ${ }^{1}$, J. Vincent ${ }^{1}$, P. Parneix ${ }^{1}$, C. Falvo ${ }^{1}$ \\ and Ph. Bréchignac ${ }^{1}$
}

\begin{abstract}
The infrared fluorescence decay and the dispersed emission spectrum are presented for gaseous benzene following $193 \mathrm{~nm}$ laser excitation. They were measured with FIREFLY (Fluorescence in the InfraRed from Excited FLYing molecules), a new home-made spectrometer. Redshift and redtail in the $\mathrm{CH}$ stretch emission spectra $(3.3 \mu \mathrm{m}$ region) demonstrate that anharmonicity plays a key role when dealing with high internal energies, as it is the case in the interstellar medium.
\end{abstract}

\section{Introduction}

Aromatic Infrared Bands (AIBs) have been observed for the very first time by Gillett et al. (1973) in the mid-infrared (IR) spectral range. Since then, Polycyclic Aromatic Hydrocarbons (PAHs) have been investigated as plausible carriers for these bands (Allamandola et al. 1985; Joblin \& Tielens 2011; Léger \& Puget 1984). Thanks to satellites such as IRAS, ISO, Spitzer, Akari and earth-bound or airborne instruments (Giard et al. 1989; Jourdain de Muizon et al. 1986), numerous observations of AIBs have been reported. AIBs are mainly found at $3.3 \mu \mathrm{m}$, $6.2 \mu \mathrm{m}$, "7.7" $\mu \mathrm{m}, 8.6 \mu \mathrm{m}, 11.3 \mu \mathrm{m}$ and $12.7 \mu \mathrm{m}$ in various environments, from diffuse clouds to stellar systems. Based on their position and shape, the AIBs can be separated in three main classes A, B and C (Peeters et al. 2002), which reveal different families of carriers with different growth scenarios and evolutionary links (Acke et al. 2010; Carpentier et al. 2012; Pino et al. 2008; Sloan et al. 2007; Carpentier et al. elsewhere in this volume). The important differences between the classes are in the $6.2 \mu \mathrm{m}$ and "7.7" $\mu \mathrm{m}$ positions (redshifted from class A to C)

\footnotetext{
${ }^{1}$ Institut des Sciences Moléculaires d'Orsay, Université Paris-Sud 11, France

${ }^{2}$ Laboratoire de Physique des Lasers, Atomes et Molécules, Université de Lille 1, France

${ }^{3}$ Institut d'Astrophysique Spatiale, Atomes et Molécules, Université Paris-Sud 11, France 
and in the type of observed object. Ultraviolet (UV) photon absorption can lead to different competing processes such as ionisation, desorption, fragmentation and infrared emission. In this paper we will focus on the latter, also called infrared fluorescence. This long lasting phenomenon (a few seconds) challenges modelling (Basire et al. 2008; Cook \& Saykally 1998; Puget \& Léger 1989) as well as gas phase experiments (Brenner \& Barker 1992; Cherchneff \& Barker 1989; Cook \& Saykally 1998; Cook et al. 1998; Shan et al. 1991; Williams \& Leone 1995). We present the IR fluorescence decay and the dispersed emission spectrum (in the $\mathrm{CH}$ stretch region around $3.3 \mu \mathrm{m}$ ) of benzene obtained with a new home-made spectrometer. We choose to begin with the benzene molecule in gas phase as a well-studied prototype and a building block of the PAH family.

\section{Experiment}

A spectrometer dedicated to measuring weak IR emission signals from photoexcited gas phase molecules, FIREFLY (Fluorescence in the InfraRed from Excited FLYing molecules), has been built (Fig. 1) as part of the Nanograins project at the Institut des Sciences Moléculaires d'Orsay (France). The instrument is at room temperature and atmospheric pressure, and is coupled to a cell containing flowing benzene (Sigma-Aldrich, purity $\geq 99 \%$ ) from a liquid reservoir maintained under low pressure and at ambiant temperature. The flow of molecules is controlled by a dosing valve. It forms an effusive flow through a $4 \mathrm{~mm}$ diameter hole and the residual pressure in the cell is around 0.2 mbar. An argon flow is used to protect the $\mathrm{CaF}_{2}$ entrance window of the cell from soot formation under laser irradiation. This implies that argon pressure has to be higher than the benzene one (>1 mbar). The benzene-argon collisional rate is estimated to $1.510^{7} \mathrm{~s}^{-1}$ and the benzene-benzene collisional rate $710^{5} \mathrm{~s}^{-1}$.

A nanosecond ArF excimer laser beam (Neweks) photoexcites gaseous benzene at $193 \mathrm{~nm}\left(51800 \mathrm{~cm}^{-1}\right)$. The fluence is kept lower than $3 \mathrm{~mJ} /$ pulse to avoid multiphoton effects and the repetition rate is set to $100 \mathrm{~Hz}$. The UV beam is directed perpendicularly to the infrared spectrometer general axis in order to avoid damages by the energetic photons. As no collection optics is used to couple the emission region with the spectrometer, only a small solid angle $(\approx 0.05$ steradians i.e., $0.4 \%$ ) of the isotropic IR emission is detected, optimized for this instrument.

The main elements of the instrument are the narrow bandpass interferential Circular Variable Filters (CVFs) replicated from the ISOCAM instrument (Cesarsky et al. 1996). They consist of three segments, covering the $2.45 \mu \mathrm{m}$ $\left(4073 \mathrm{~cm}^{-1}\right)$ to $14.5 \mu \mathrm{m}\left(690 \mathrm{~cm}^{-1}\right)$ spectral range. In this experiment, only the $2.45 \mu \mathrm{m}\left(4073 \mathrm{~cm}^{-1}\right)$ to $4.55 \mu \mathrm{m}\left(2195 \mathrm{~cm}^{-1}\right)$ segment is used in order to probe the $\mathrm{CH}$ stretch region. They have a high transmission $(75 \%$ at $3.3 \mu \mathrm{m})$ and a moderate resolving power $(\lambda / \delta \lambda=45$ at $3.3 \mu \mathrm{m})$. They are fixed on a computercontrolled rotatable wheel. Specific ellipsoid mirrors have been designed to shape the IR beam to the desired dimensions. They are gold-coated without any additional protection, so that the reflection coefficient is at least $97-98 \%$ in the 2-20 $\mu \mathrm{m}$ spectral range. The first mirror focuses the beam on the filter with a 


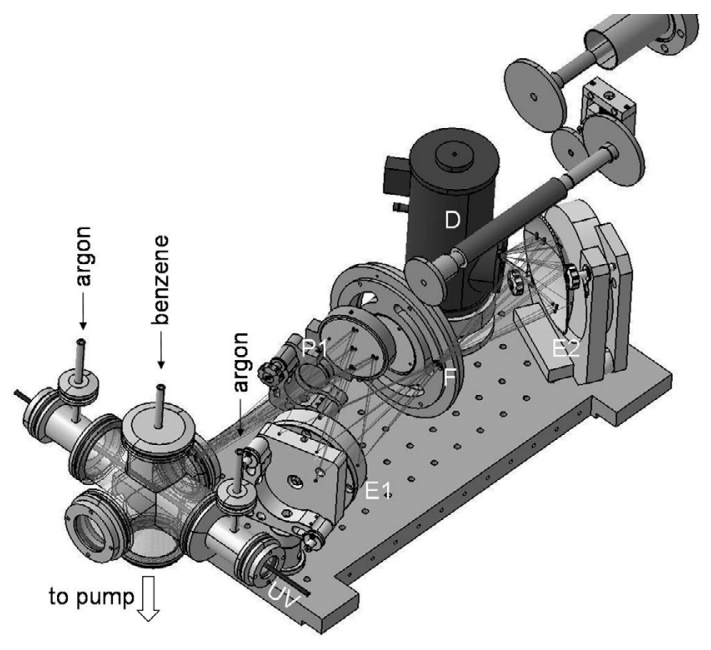

Fig. 1. Scheme of the infrared emission spectrometer. The cell with benzene and argon tubes is represented (left). The UV beam crosses the cell (lower left). The IR beam is represented by multiple grey lines. A plane mirror (P1) reflects the light on a first ellipsoid mirror (E1) which focuses the beam on the rotatable wheel containing the $2.45 \mu \mathrm{m}$ $\left(4073 \mathrm{~cm}^{-1}\right)$ to $4.55 \mu \mathrm{m}\left(2195 \mathrm{~cm}^{-1}\right)$ bandpass filter $(\mathrm{F})$. It diverges and a second ellipsoid mirror (E2) focuses the beam on the InSb detector (D).

rectangular cross section of $2.5 \times 7.5 \mathrm{~mm}^{2}$. A second mirror shapes again the beam into a $2 \times 2 \mathrm{~mm}^{2}$ square cross section optimized for a nitrogen cooled InSb detector (Infrared Associates). The signal is then amplified through a $1.5 \mathrm{~Hz}-20 \mathrm{kHz}$ preamplifier before being digitized and recorded via a computer. The instrument was spectrally calibrated using gaseous ethanol, benzene and $\mathrm{CO}_{2}$ absorptions with, in this case, a $873 \mathrm{~K}$ blackbody radiation as an IR source.

\section{Results and discussion}

Benzene has six $\mathrm{CH}$ stretching modes including two IR active ones. After electronic absorption (cross section at $193 \mathrm{~nm} \approx 2010^{-18} \mathrm{~cm}^{2}$ as reported by Suto et al. 1992), several non radiative and radiative processes occur. Successive internal conversions convert the initial electronic excitation in the $\mathrm{S}_{2}$ state $\left({ }^{1} \mathrm{~B}_{1 u}\right)$ into ground state high vibrational excitation, accompanied by Intramolecular Vibrational energy Redistribution (IVR - for a review, see Nesbitt \& Field 1996) and followed by the emission of IR photons forming a long-lasting cascade. Figure 2a shows the infrared fluorescence decay following $193 \mathrm{~nm}$ excitation at $\mathrm{t}=0$, with a $10^{-8} \mathrm{~S}$ resolution. No interferential filters are used (the wavelength range is therefore that one of the InSb detector i.e., 2.5 to $5.5 \mu \mathrm{m}$ ) and the signal is averaged over 300 laser shots. The initial rise of $\approx 4-5 \mu$ s is due to the response time of the detector and $1.5 \mathrm{~Hz}-20 \mathrm{kHz}$ preamplifier. The decay fits well with a biexponential function with characteristic times of $8 \mu \mathrm{s}$ and $135 \mu \mathrm{s}$. A single exponential 

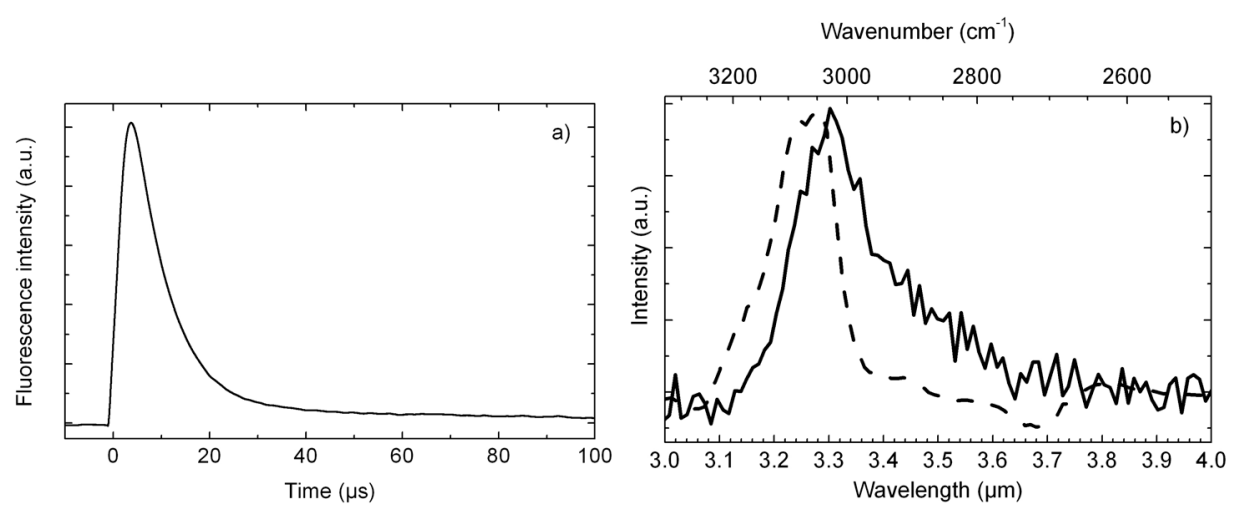

Fig. 2. a) Total fluorescence decay ( $\mathrm{CH}$ stretch region) following $193 \mathrm{~nm}$ excitation for gas phase benzene. $t=0$ stands for the UV excitation. b) Dispersed IR fluorescence as a function of the wavelength (CH stretch region) following $193 \mathrm{~nm}$ excitation for gas phase benzene. Dashed line: benzene absorption (300 K, $\approx 90 \mathrm{mbar}$ ) recorded with the same spectrometer. The wavelength step is $0.01 \mu \mathrm{m}$.

decay is not expected and the precise modeling of Barker \& Golden (1984) and Yerram et al. (1990) is preferred and briefly discussed hereafter. Such a fit gives only access to a rough evaluation of the cooling rate. The spectrally resolved IR fluorescence in the $\mathrm{CH}$ stretch region is shown in Figure $2 \mathrm{~b}$. It was obtained by integrating the time-resolved IR signal through the 0-100 $\mu$ s windows for each wavelength from $3 \mu \mathrm{m}\left(3333 \mathrm{~cm}^{-1}\right)$ to $4 \mu \mathrm{m}\left(2500 \mathrm{~cm}^{-1}\right)$ with a $0.01 \mu \mathrm{m}$ step. A noticeable redtail is observed in the spectrum up to $4 \mu \mathrm{m}$. Redshift and redtail are the signatures of anharmonicity in the ground electronic state. The presented spectrum compares very well with that of Shan et al. (1991) (infrared emission from excited benzene at $193 \mathrm{~nm}$ in a cell), although ours seems to be slightly wider. Benzene IR absorption at $300 \mathrm{~K}$ is also shown in Figure $2 \mathrm{~b}$ (dashed line). The IR source is a $873 \mathrm{~K}$ blackbody radiation and FIREFLY was used with a lock-in amplifier to record the spectrum. The emission and the absorption bands differ in position, width and asymmetry. The first moment of the emission band located at $2994 \mathrm{~cm}^{-1}$ is $74 \mathrm{~cm}^{-1}$ redshifted from the first moment of the absorption band located at $3068 \mathrm{~cm}^{-1}$ and the width of the emission band is significantly larger than the absorption band (Fig. 2b). These differences between absorption and emission clearly show the preponderant role of the internal energy. Williams \& Leone (1995) and Cook et al. (1998) measured the naphthalene IR emission for two different excitation energies and showed that the higher the internal energy, the stronger the redshift and redtail are, as a consequence of the anharmonicity.

IR emission cascades measured in the laboratory are faster than interstellar ones. This is mainly because the collisional quenching, only present in the experiment, opens a new competing relaxation pathway. Barker \& Golden (1984) and Yerram et al. (1990) quantified the internal energy loss per molecular collision as a function of the internal energy by measuring IR fluorescence rates. They found 
that collisions between excited benzene and unexcited benzene efficiently reduce the internal energy (by about $1500 \mathrm{~cm}^{-1}$ per collision at an internal energy of $40000 \mathrm{~cm}^{-1}$ ). Given our collisional rate, the order of magnitude of internal energy loss is $10000 \mathrm{~cm}^{-1}$ in $10 \mu \mathrm{s}$. Another competing pathway is the benzene dissociation. Tsai et al. (2000) and Kislov et al. (2004) measured and calculated that the values of the benzene lifetime at $193 \mathrm{~nm}$ are respectively 10 and $11 \mu \mathrm{s}$ and that the branching ratio for H-loss is $99.6 \%$. One could wonder to what extent the phenyl radical $\mathrm{C}_{6} \mathrm{H}_{5}$ contributes to the recorded emission: the $\mathrm{CH}$ bond clivage costs $4.9 \mathrm{eV}$ (Davico et al. 1995), so the phenyl product internal energy is less than $1.5 \mathrm{eV}(6.4 \mathrm{eV}-4.9 \mathrm{eV})$; because such internal energy is somehow comparable to the $\mathrm{CH}$ stretch frequency $(0.4 \mathrm{eV})$, radiative relaxation through that channel is not favoured. Moreover, the absolute phenyl $\mathrm{CH}$ stretch $\left(3071 \mathrm{~cm}^{-1}\right)$ absorption intensity is smaller than the benzene $\mathrm{CH}$ stretch absorption intensity (Friderichsen et al. 2001; Spedding \& Whiffen 1956). Therefore, no strong emission from the phenyl radical is expected. Due to collisional quenching, the benzene dissociation is less and less likely and only the competition between IR emission and collisions remains.

\section{Conclusion}

The IR fluorescence decay and the spectrally resolved emission spectrum are presented for gaseous benzene excited at $193 \mathrm{~nm}$, highlighting a large anharmonicity. Spectroscopic differences between absorption and emission spectra underline the necessity to compare astrophysical emission data not only with laboratory absorption spectra but also with emission spectra, under conditions similar to those encountered in space. As mentioned in the introduction, benzene represents a prototype molecule, showing a broader emission spectrum than the $3.3 \mu \mathrm{m}$ AIB which typically has a $30 \mathrm{~cm}^{-1}$ FWHM. As shown for example by Shan et al. (1991), for increasingly larger PAHs, the emission band becomes narrower and similar to the AIBs. Due to experimental limitations, coronene is the biggest PAH measured in emission (Cook et al. 1996). The interstellar emitters should however not exceed a size of a few hundreds carbon atoms, in order to reach the temperatures of $\sim 1000 \mathrm{~K}$ that are required to emit at $3.3 \mu \mathrm{m}$. More generally, IR emission measurements are essential tools to improve our understanding of molecules. Observations, theoretical and experimental research need to continue their joined effort to shed new light on the AIB carriers.

The authors wish to thank L. Vigroux for giving them the opportunity to use the CVF filters. This work was in part supported by the French CNRS-INSU national program "Physique et Chimie du Milieu Interstellaire" (PCMI) and by the French Agence Nationale de la Recherche under the project grant ANR-10-BLAN-0501-GASPARIM.

\section{References}

Acke, B., Bouwman, J., Juhsz, A., et al., 2010, ApJ, 718, 558

Allamandola, L.J., Tielens, A.G.G.M., \& Barker, J.R., 1985, Astrophys. J. Lett., 290, L25 
Barker, J.R., \& Golden, R.E., 1984, J. Phys. Chem., 88, 1012

Basire, M., Parneix, P., \& Calvo, F., 2008, J. Chem. Phys., 129, 081101

Brenner, J., \& Barker, J.R., 1992, ApJ, 388, L39

Carpentier, Y., Féraud, G., Dartois, E., et al., 2012, A\&A, 548, A40

Cesarsky, C. J., Abergel, A., Agnese, P., et al., 1996, A\&A, 315, L32

Cherchneff, I., \& Barker, J.R., 1989, ApJ, 341, L21

Cook, D.J., \& Saykally, R.J., 1998, ApJ, 493, 793

Cook, D.J., Schlemmer, S., Balucani, N., et al., 1998, J. Phys. Chem. A, 102, 1465

Cook, D.J., Schlemmer, S., Balucani, N., et al., 1996, Nature, 380, 227

Davico, G.E., Bierbaum, V.M., DePuy, C.H., Ellison, G.B., \& Squires, R.R., 1995, J. Amer. Chem. Soc., 117, 2590

Friderichsen, A.V., Radziszewski, J.G., Nimlos, M.R., et al., 2001, J. Amer. Chem. Soc., 123, 1977

Giard, M., Serra, G., Caux, E., Pajot, F., \& Lamarre, J.M., 1989, A\&A, 215, 92

Gillett, F.C., Forrest, W.J., \& Merrill, K.M., 1973, ApJ, 183, 87

Joblin, C., \& Tielens, A. (eds.), 2011, PAHs and the Universe: A Symposium to Celebrate the 25th Anniversary of the PAH Hypothesis, 46

Jourdain de Muizon, M., Geballe, T.R., D'Hendecourt, L.B., \& Baas, F., 1986, Astrophys. J. Lett., 306, L105

Kislov, V.V., Nguyen, T.L., Mebel, A.M., Lin, S.H., \& Smith, S.C., 2004, J. Chem. Phys., 120,7008

Léger, A., \& Puget, J.L., 1984, A\&A, 137, L5

Nesbitt, D.J., \& Field, R.W., 1996, J. Phys. Chem., 100, 12735

Peeters, E., Hony, S., Kerckhoven, C.V., et al., 2002, A\&A, 390, 1089

Pino, T., Cao, A.T., Carpentier, Y., Dartois, E., D'Hendecourt, L., \& Bréchignac, P., 2008, A\&A, 251, 393

Puget, J.L., \& Léger, A., 1989, ARA\&A, 27, 161

Shan, J., Suton, M., \& Lee, L.C., 1991, ApJ, 383, 459

Sloan, G.C., Jura, M., Duley, W.W., et al., 2007, ApJ, 664, 1144

Spedding, H., \& Whiffen, D.H., 1956, Proc. Royal Soc. A, 238, 245

Suto, M., Wang, X., Shan, J., \& Lee, L., 1992, J. Quant. Spectr. Rad. Transfer, 48, 79

Tsai, S.-T., Lin, C.-K., Lee, Y. T., \& Ni, C.-K., 2000, J. Chem. Phys., 113, 67

Williams, R.M., \& Leone, S.R., 1995, ApJ, 443, 675

Yerram, M.L., Brenner, J.D., King, K.D., \& Barker, J.R., 1990, J. Phys. Chem., 94, 6341 